\title{
OBSTRUÇÃO GASTROINTESTINAL POR FITOBEZOAR NA CIRURGIA BARIÁTRICA
}

\section{GASTROINTESTINAL OBSTRUCTION BY PHYTOBEZOAR AFTER BARIATRIC SURGERY}

\author{
Álvaro Antônio Bandeira Ferraz, TCBC-PE ${ }^{1}$; Vladimir Curvêlo Tavares de Sá²; \\ Pedro Carlos Loureiro de Arruda ${ }^{3}$; Cristiano de Souza Leã $0^{4}$; \\ Josemberg Marins Campos ${ }^{5}$; Edmundo Machado Ferraz, TCBC-PE ${ }^{6}$
}

\begin{abstract}
RESUMO: Objetivo: O objetivo do presente estudo foi avaliar o quadro clínico, a incidência, os fatores predisponentes e a evolução de pacientes com fitobezoar após Gastroplastia Vertical e Y de Roux. Método: No período de Novembro de 1997 à Janeiro de 2004, foram realizadas 512 operações para o tratamento da Obesidade mórbida, seguindo a técnica proposta por Fobi/Capella (Septação gástrica com reconstituição em Y de Roux Proximal). Em dez pacientes foram identificados fitobezoar. Resultados: A incidência de fitobezoar, causando algum tipo de obstrução gastrointestinal, foi de 1,95\% (10/512). Todos os casos de fitobezoar estavam relacionados ao fio de sutura inabsorvível do tipo prolene. Sete pacientes apresentaram quadro semioclusivo e de obstrução intestinal e foram tratados com laparotomia exploradora, ressecção da anastomose enteroenteral e confecção de nova enteroenteroanastomose. Nos três pacientes com bezoar situado na anastomose gastrojejunal, a secção do fio e a retirada do corpo estranho foram realizadas por endoscopia digestiva alta. Conclusão: A confecção de anastomose intestinal com fio inabsorvível predispõe a formação de bezoar em pós-operatório de gastroplastia (Rev. Col. Bras. Cir. 2006; 33(1): 35-38).
\end{abstract}

Descritores: Bezoares; Gastroplastia; Obesidade; Obesidade/cirurgia; Obstrução intestinal.

\section{INTRODUÇÃO}

Os bezoares são classificados de acordo com a sua composição em fitobezoar (fibra vegetal), tricobezoar (cabelo), lactobezoar (compostos lácticos), entre outros ${ }^{1-4}$. Geralmente o fitobezoar, é formado por sementes, raízes, cascas, fibras, celulose, tanina, liguina e derivados de frutas e vegetais, como abacaxi e caqui ${ }^{1,5,6}$.

$\mathrm{O}$ fitobezoar é o mais freqüente, correspondendo à cerca de $40 \%$ dos bezoares, especialmente nos pacientes submetidos à cirurgia gástrica ${ }^{6,7,8}$.

A cirurgia gástrica prévia é o principal fator predisponente na formação do fitobezoar, mas a mastigação inadequada, dietas à base de fibras não digeríveis e alterações na motilidade gástrica também influenciam na sua formação ${ }^{9}$.

O aumento do número de casos de obesidade mórbida tratados cirurgicamente vem crescendo de maneira exponencial. O número de cirurgias bariátricas realizadas em todo o mundo cresceu essencialmente baseados em dois fatores: os bons resultados do tratamento cirúrgico e do crescimento significativo dos casos de obesidade ${ }^{10,11}$.
Com a crescente freqüência de cirurgias gástricas realizadas principalmente no tratamento da obesidade mórbi$\mathrm{da}$, associado às modificações dietéticas impostas por este tipo de cirurgia, houve um aumento significativo da predisposição à formação de fitobezoar.

Objetiva-se neste trabalho avaliar o quadro clínico, a incidência, os fatores predisponentes e a evolução de pacientes com fitobezoar após Gastroplastia Vertical em Y de Roux.

\section{MÉTODO}

No período de Novembro de 1997 à Janeiro de 2004, foram realizadas 512 operações para o tratamento da Obesidade mórbida, seguindo a técnica proposta por Fobi/ Capella $^{12,13}$ (Septação gástrica com reconstituição em Y de Roux Proximal).

A idade variou entre 20 e 59 anos de idade (média de 34 anos). Em relação ao sexo, 313 pacientes eram do sexo feminino, e 199 do sexo masculino.

O peso médio apresentado foi $146 \mathrm{Kg}$, variando de 97 a $287 \mathrm{Kg}$. A altura variou entre 1,48 a 1,90 metros, com uma média de 1,65 metros.

1. Professor Adjunto do Departamento de Cirurgia da Universidade Federal de Pernambuco (UFPE); Mestre e Doutor em Medicina pela UFPE; Especialização em Infecção em Cirurgia no Medical College of Wisconsin - EUA; Pós-Doutorado em Transplante de Fígado na Universidade de Miami - EUA; Professor Livre-Docente da Universidade de São Paulo - Ribeirão Preto.

2. Médico Residente de $5^{\circ}$ ano do Serviço de Cirurgia Geral do HC da UFPE.

3. Professor do Departamento de Cirurgia da UFPE.

4. Professor Substituto do Departamento de Cirurgia da UFPE; Aluno da Pós-graduação em Cirurgia da UFPE - nível Doutorado.

5. Mestre e Doutor em Cirurgia pela Universidade Federal de Pernambuco.

6. Professor Titular de Cirurgia Abdominal e Bases da Técnica Cirúrgica da UFPE; Chefe do Serviço de Cirurgia Geral do Hospital das Clínicas da UFPE; Doutor e Livre Docente pela UFPE; Fellow do American College of Surgeons (FACS) e do Surgical Infection Society.

Recebido em 02/09/2005

Aceito para publicação em 04/11/2005

Conflito de interesses: nenhum

Fonte de financiamento: nenhuma

Trabalho realizado no Departamento de Cirurgia da Universidade Federal de Pernambuco (UFPE). 
Os pacientes atingiram um IMC médio de $46 \mathrm{Kg} / \mathrm{m}^{2}$, com limites entre 35 a $98 \mathrm{Kg} / \mathrm{m}^{2}$.

Os critérios utilizados para a indicação cirúrgica foram baseados nas determinações do "National Institutes of Health Consensus Development Panel on Gastrointestinal Surgery for Severe Obesity" "14 que incluem um IMC maior de $40 \mathrm{Kg} / \mathrm{m}^{2}$ ou IMC maior que $35 \mathrm{Kg} / \mathrm{m}^{2}$ com comorbidades severas.

A avaliação pré-operatória englobou além de exames hematológicos e bioquímicos, ultra-sonografia de abdome total, endoscopia digestiva alta, avaliações endocrinológicas, cardiológicas, pneumológica, psíquica, nutricional, ortopédica e da equipe de enfermagem.

Os critérios de exclusão do protocolo foram: idade acima de 60 anos, gravidez, obesidade decorrente de certos distúrbios psiquiátricos e endocrinológicos, dependência química e doenças associadas relacionadas a um elevado risco cirúrgico ${ }^{15}$.

A cirurgia bariátrica realizada foi a descrita por Fobi ${ }^{12}$ e posteriormente modificada por Capella ${ }^{13}$. Neste tipo de cirurgia algumas etapas foram padronizadas e sistematizadas. A realização do tubo gástrico de $50 \mathrm{ml}$ com grampeador linear era seguida de uma sutura externa, hemostática, na linha de grampeamento com fio de Vycril 3-0®. A gastroenteroanastomose foi realizada em dois planos, sendo o primeiro plano.(interno) com fio de Vycril 3-0® e o segundo plano (externo) com fio de $0 \AA$ Prolene 3-0®. Após a realização de uma enteroenteroanastomose com grampeador linear uma sutura intraluminar, com fio de Prolene 3-0®, em toda a extensão da linha de grampeamento foi realizada. Esta sutura teve objetivo hemostático.

Após a intervenção operatória, os pacientes foram encaminhados à Unidade de Cuidados intermediários (UCI) onde permaneciam por $12 \mathrm{~h}$. A dieta foi iniciada em torno do $2^{\circ}$ dia pós-operatório (DPO) e a alta, em geral, ocorreu no $3^{\circ} \mathrm{ou}$ $4^{\circ} \mathrm{DPO}$.

O acompanhamento ambulatorial se fez com visitas a partir da $2^{\circ}$ semana do pós-operatório e com 1, 2, 3, 6, 12, 18 e 24 meses, no ambulatório de Cirurgia Geral concomitantemente com o Serviço de Endocrinologia e Psicologia.

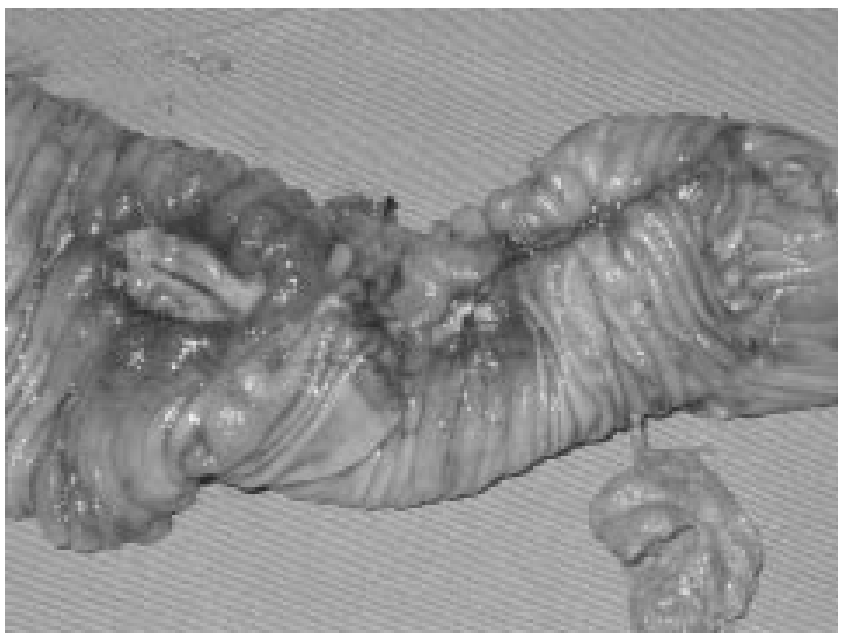

Figura 1 - Fitobezoar fixo ao fio de sutura. Notar ulceração no local da fixação do fitobezoar.

\section{RESULTADOS}

Em dez pacientes foi identificado fitobezoar. Foram nove pacientes do sexo feminino e um do sexo masculino com idade variando de 42 a 60 anos, com IMC pré gastroplastia de 43 a 52.

A incidência de fitobezoar com sinais de semioclusão ou de obstrução gastrointestinal foi de 1,95\% (10/512), estando sempre associada à presença de fio de sutura inabsorvível (Prolene 3-0 ${ }^{\circledR}$ ) e úlcera de tamanho variável, recobertas por fibrina e de bordas elevadas (Figuras 1 e 2). Não houve mortalidade nos 10 pacientes, que tiveram permanência hospitalar média de 4,3 dias.

\section{Sintomatologia:}

Dois pacientes apresentaram quadro agudo de obstrução intestinal.

Cinco pacientes apresentaram quadro de semioclusão intestinal que evoluiu em um período de três a seis meses. Durante este período os pacientes alternavam períodos de sintomatologia intensa, com dor e distensão abdominal, vômitos e cólicas, com períodos assintomáticos. Diversos exames não evidenciavam a causa, como endoscopias, trânsitos intestinais, tomografias abdominais. Como os eventos de semioclusão se tornavam freqüentes, sendo inclusive necessários internamentos para resolução clínica, as pacientes foram levados, eletivamente, a laparotomias exploradoras.

Os sete pacientes com quadro semioclusivo e de obstrução intestinal foram tratados com laparotomia exploradora e ressecção da anastomose enteroenteral e reconfecção desta anastomose. Em uma pacientes foi realizada uma enterotomia, retirada do fitobezoar e do fio de sutura e enterorrafia (Figura 3).

Em três pacientes a sintomatologia apresentada foi de uma obstrução alta com náuseas e vômitos pós-prandiais. Em duas pacientes o fitobezoar gástrico estava associado com úlcera de boca anastomótica. Estes pacientes foram tratados satisfatoriamente por procedimento endoscópico de retirada do fitobezoar e do fio de sutura exposto.

Os pacientes se encontram assintomáticos desde a retirada do bezoar, até a presente data.

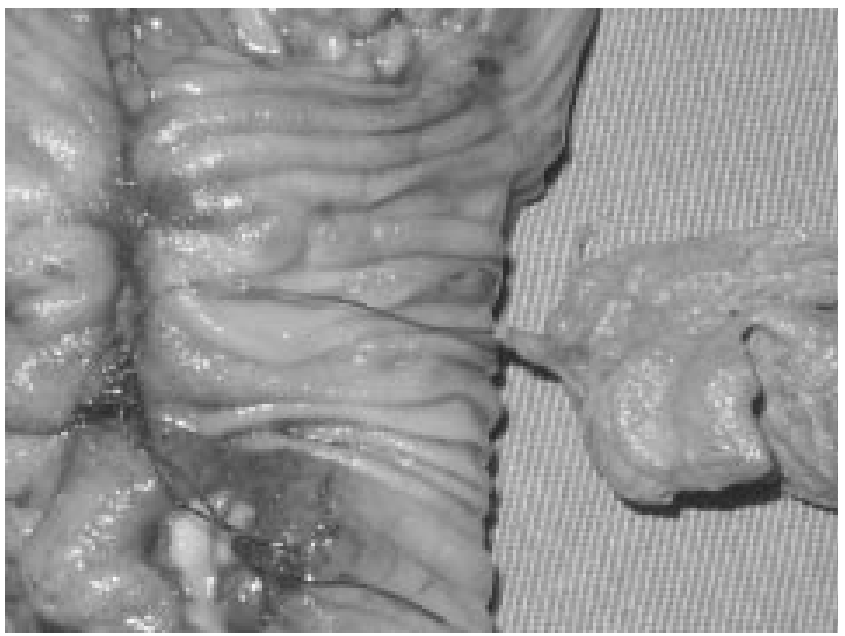

Figura 2 - Imagem aproximada do fitobezoar fixo ao fio de sutura. 


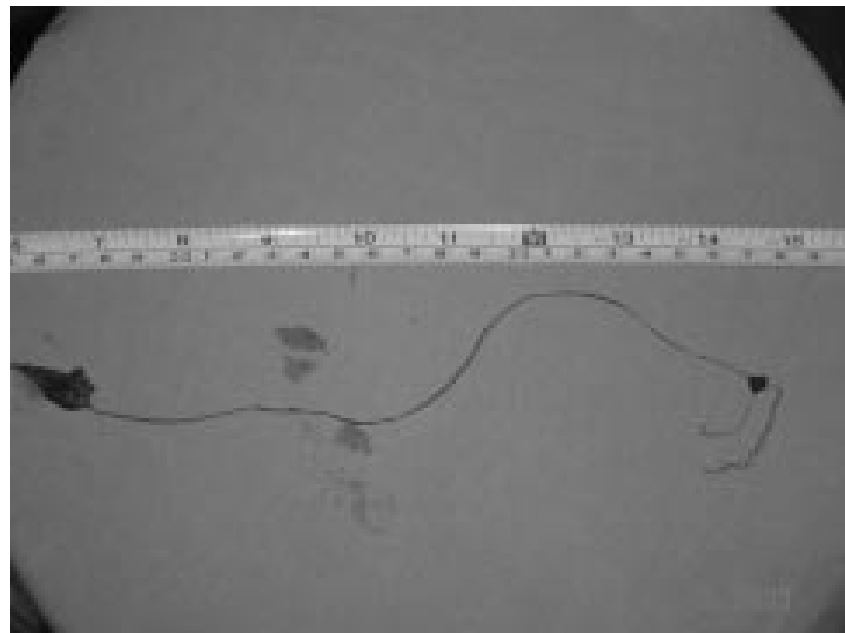

Figura 3 - Fitobezoar fixo ao fio de sutura e funcionando com êmbolo.

\section{COMENTÁRIOS}

O paciente portador de obesidade mórbida tratado cirurgicamente é acompanhado regularmente pelo cirurgião, pelo endocrinologista, pelo psicólogo e por um especialista em nutrição como intuito de modificar o estilo de vida e principalmente seus hábitos alimentares. Neste período, o paciente é estimulado a modificar seu hábito alimentar com especial atenção à ingesta de fibras, frutas e proteínas.

A operação gástrica associada a uma dieta rica em fibras inabsorvíveis ou de difícil absorção são fatores predisponentes significativos para a formação do fitobezoar ${ }^{1,5,6,9}$. Concomitante a estes fatores de risco a presença de um fio de sutura não absorvível, funcionando como âncora para este fitobezoar, chegamos a uma incidência de 1,95\% pósgastroplastias.

Em nossa casuística a utilização de fios de sutura inabsorvíveis, do tipo Prolene ${ }^{\circledR}$, em sutura intraluminar, foi o fator primordial na etiologia do fitobezoar. Este tipo de sutura,envolvendo a linha de grampos (intraluminar) tem o objetivo de realizar a hemostasia e é rotineiramente utilizada.
A sintomatologia variou essencialmente de acordo com o nível da impactação. Nos pacientes em que o fitobezoar se localizou na anastomose gastrojejunal o quadro clínico apresentado foi de náusea, vômito, dor epigástrica e azia. Nas obstruções jejunais o quadro de distensão abdominal, dor abdominal tipo cólica e vômitos fecalóides.

A tomografia de abdome e o estudo radiológico contrastado não diagnosticaram o nível da obstrução intestinal, mesmo sendo revisados no pós-operatório por radiologistas e cirurgiões, que conheciam o achado cirúrgico.

Nos casos em que o bezoar esteve localizado na anastomose gastrojejunal o tratamento endoscópico foi efetivo. Tem sido descrito uso de soluções a base de glicol-eletrólitos de polietileno ou manitol no tratamento de quadros semi-oclusivos do intestino ${ }^{6,16}$. No estudo atual, não foi indicado tal tratamento devido a ausência de diagnóstico específico no pré-operatório.

$\mathrm{O}$ bezoar de intestino delgado pode ser tratado sem enterotomia $^{6}$. No entanto, a fragmentação manual, durante a laparotomia foi sempre difícil e incapaz de fragmentar por completo o bezoar. Além disto, há a necessidade de se retirar o fio inabsorvível da anastomose, com o intuito de evitar recorrências.

As úlceras associadas ao bezoar não devem ser tratadas, pois a simples retirada do mesmo acarretará na sua cicatrização espontânea ${ }^{6}$.

Não foi diagnosticado nenhum caso de fitobezoar nos pacientes submetidos a cirurgias após Janeiro de 2004, fato que coincide com a utilização de fio absorvíveis (monocryl) nas suturas intraluminares.

É importante notar a forte relação do fitobezoar com o fio de sutura utilizado. O fio inabsorvível utilizado serviu de "âncora" para o fitobezoar e em cinco pacientes funcionou como êmbolo determinando quadros de semi-oclusão intestinal intermitente que muitas vezes cedia inteiramente para recomeçar dias ou semanas após.

Nosso estudo evidenciou que a confecção de anastomose intestinal com fio inabsorvível contribui para formação de bezoar nos pacientes submetidos ao tratamento cirúrgico para obesidade mórbida.

\begin{abstract}
Background: The aim of this study was to evaluate the relation, incidence, clinical features, predisposing factors and phytobezoar in patients submitted to bariatric surgeries. Methods: During November 1997 and January 2004, 512 bariatric surgeries (Fobi/Capella bypass) were performed to treat morbid obesity. In 10 patients phytobezoar was identified. Results: The incidence of phytobezoar resulting in gastrointestinal obstruction was 1,95\% (10/512). All cases of phytobezoar were related to unabsorbed suture stitch (prolene). In seven patients an intestinal semi-occlusive or total intestinal obstruction was treated by laparotomy, resection of the anastomosis and confection of a new enteroanastomosis. In three patients the bezoars were located in the gastrojejunal anastomosis and was successfully treated by upper endoscopic procedure. Conclusion: The use of unabsorbed suture stitch predicted the formation of phytobezoar in the late postoperative period of gastroplasty patients.
\end{abstract}

Key words: Phytobezoars; Gastroplasty; Obesity; Obesity/surgery; Intestinal obstruction.

\section{REFERÊNCIAS}

01. Erzurumlu K, Malazgirt Z, Bektas A, et al. Gastrointestinal bezoars: a retrospective analysis of 34 cases. World J Gastroenterol. 2005;11(12):1813-7.
02. Andrus CH, Ponsky JL. Bezoars: classification, pathophysiology, and treatment. Am J Gastroenterol. 1988;83(5):476-8.

03. Saeed ZA, Rabassa AA, Anand BS. An endoscopic method for removal of duodenal phytobezoars. Gastrointest Endosc. 1995;41(1):74-6. 
04. Gurses N, Gurses N, Ozkan K, et al. Bezoars: analysis of seven cases. Z Kinder Chir. 1987;42(5):291-2.

05. Hayes PG, Rotstein OD. Gastrointestinal phytobezoars: presentation and management. Can J Surg. 1986;29(6):419-20.

06. Coelho JCU, Gonçalves CG, Madureira Filho D. Bezoar. In: Coelho JCU. Aparelho digestivo: clínica e cirurgia. 3ed. São Paulo: Atheneu; 2004. p. 595-599.

07. Chisholm EM, Leong HT, Chung SC, et al. Phytobezoar: an uncommon cause of small bowel obstruction. Ann R Coll Surg Engl. 1992;74(5):342-4.

08. Schwab KS, Cheng EH. "Postgastrectomy" bezoar secondary to gastric cancer. J Clin Gastroenterol. 1993;16(1):45-7.

09. Escamilla C, Robles-Campos R, Parrilla Paricio P, et al. Intestinal obstruction and bezoars. J Am Coll Surg.1994;179(3):285-8.

10. Deitel M. Overweight and obesity worldwide now estimated to involve 1.7 billion people. Obes Surg. 2003;13(3):329-30.

11. Deitel M. Mounting the worldwide effort against obesity. Obes Surg. 2005;15(5):595-7.

12. Fobi MAL, Lee H, Flemming A. The surgical technique of the banded Roux in Y gastric bypass. J Obesity Weight Reg. 1989;8(1):99-102.
13. Capella RF, Capella JF, Mandac H. Vertical banded gastroplasty - Gastric bypass: preliminary report. Obes Surg. 1991;1(4):38995.

14. American Society of Bariatric Surgery. Rationale for surgery(1)1: 13 telas. Available from: http://www.asbs.org.

15. NIH Conference. Gastrointestinal surgery for severe obesity. Consensus Development Conference Panel. Ann Intern Med. 1991;115(12):956-61.

16. Shah M, Nakanishi A. Polyethylene glycol-electrolyte solution for rectal sunflower seed bezoar. Pediatr Emerg Care. 1990;6(12):127-8.

Endereço para correspondência:

Av. Beira Rio 240, Apto 2501

Madalena

50750-400 - Recife-PE

Tel.: 5581- 3227.29.91

FAX: 5581- 3271.15.26

E-mail: aabf@ truenet.com.br 